\title{
Algorithmenbasierter Handel und Kartellverbot
}

Heinemann, Andreas

Posted at the Zurich Open Repository and Archive, University of Zurich ZORA URL: https://doi.org/10.5167/uzh-212287

Book Section

Originally published at:

Heinemann, Andreas (2021). Algorithmenbasierter Handel und Kartellverbot. In: Zimmer, Daniel. Regulierung für Algorithmen und Künstliche Intelligenz. Baden-Baden: Nomos, 269-291. 


\title{
Algorithmenbasierter Handel und Kartellverbot
}

\author{
Prof. Dr. Andreas Heinemann*
}

\section{Die 'machinery question'}

Maschinen haben den Menschen von mühsamer und gefährlicher Arbeit entlastet und sind deshalb im Grundsatz positiv konnotiert, auch wenn sich gezeigt hat, dass große Anstrengungen zur Verhinderung von Unfällen und Umweltbeeinträchtigungen erforderlich sind. Seit 200 Jahren gibt es allerdings auch eine grundsätzliche Debatte über Nutzen und Nachteil von Maschinen. 1821 fügte David Ricardo der dritten Auflage seines Werks "On the Principles of Political Economy and Taxation" das aufsehenerregende Kapitel "On Machinery" hinzu, in dem er die These aufstellte, dass der Einsatz von Maschinen nachteilig für die Beschäftigung sein könne. ${ }^{1}$ Seither wird die machinery question intensiv diskutiert: Welche Wirkungen haben Mechanisierung, Maschinisierung und Automatisierung auf die Arbeitsplätze? In der digitalen Ökonomie hat die Debatte um Jobverluste und -gewinne einen neuen Höhepunkt erreicht. Die Science-Fiction-Welt geht einen Schritt weiter: Hier sind Maschinen nicht nur Killer von Arbeitsplätzen, sondern sie werden zur Bedrohung für das Überleben der Menschen oder gar der gesamten Menschheit überhaupt. Prominente Beispiele sind der Bordcomputer HAL 9000 in Stanley Kubricks "2001: Odyssee im Weltraum" und das "Verteidigungssystem" Skynet in den TerminatorFilmen.

Und nun ${ }^{2}$ hat die machinery question das Kartellrecht erreicht: Werden die Maschinen Kartelle bilden und den Wettbewerb bedrohen? Streitig wird bereits diskutiert, ob es sich hierbei um eine reale Gefahr handelt, oder ob das Thema in die zweite Kategorie fällt, also reine Science-Fiction

* Der Verfasser ist Professor an der Universität Zürich und Präsident der schweizerischen Wettbewerbskommission. Er gibt seine persönliche Meinung wieder.

1 S. z.B. die Analyse von Blaug, Economic Theory in Retrospect, 5. Aufl. 1996, S. $129 \mathrm{f}$.

2 Der (soweit ersichtlich) erste wissenschaftliche Artikel zum Algorithmen-KartellProblem datiert vom Januar 2014 und stammt von Salil Mehra, De-Humanizing Antitrust: The Rise of the Machines and the Regulation of Competition, abrufbar unter: www.researchgate.net/publication/272245466. 
ist. Die "Realisten" verweisen auf das Potential von Künstlicher Intelligenz (KI) und Machine Learning, zudem auf die Erfolge von Algorithmen und KI bei Brettspielen, Poker und Experimenten wie dem Gefangenendilemma. ${ }^{3}$ Die "Fiktionalisten" heben demgegenüber die Schwierigkeiten hervor, denen Preisabsprachen in der komplexen Wirtschaftswirklichkeit begegnen. ${ }^{4}$

Vor diesem Hintergrund soll im Folgenden anhand existierenden Fallrechts veranschaulicht werden, inwieweit Algorithmen bei der Kartellrechtsanwendung bereits Beachtung gefunden haben (II.). Auch wenn sich erweisen wird, dass der Beitrag der Algorithmen zur Verhaltenskoordination bisher bescheiden geblieben ist, lassen sich aus dem beginnenden Fallrecht erste Schlussfolgerungen ziehen. Auf einer nächsten Stufe bleibt sodann zu untersuchen, welche Grenzen das Kartellrecht selbständigeren Formen algorithmischer Koordination zieht (III.). Es liegen Vorschläge vor, die auf teilweise radikale Weise einen Umbau des Kartellrechts verlangen. Das Fazit enthält eine Bestandsaufnahme geklärter und offener Fragen im Zusammenhang mit algorithmischer Kollusion (IV.). 5

3 S. z.B. Ezrachi/Stucke, Virtual Competition - The Promise and Perils of the Algorithm-Driven Economy, 2016; die Autoren warnen insbesondere davor, dass die unsichtbare Hand des Wettbewerbs durch die digitale Hand der Algorithmen abgelöst werden könnte (ibid., S. viii); Calvano/Calzolari/Denicolò/Pastorello, Algorithmic Collusion: A Real Problem for Competition Policy?, CPI Antitrust Chronicle July 2020, S. 28; Klein, (Mis)Understanding Algorithmic Collusion, CPI Antitrust Chronicle July 2020, S. 53.

4 S. z.B. Petit, Antitrust and Artificial Intelligence: A Research Agenda, 8 Journal of European Competition Law \& Practice 361-2 (2017); Schwalbe, Algorithms, Machine Learning, and Collusion, 14 Journal of Competition Law \& Economics 568, 600 (2018): "Given that the problem of algorithmic collusion currently seems to belong to the realm of legal sci-fi, it seems advisable to devote the limited resources of competition authorities to more pressing competitive concerns than highly speculative conjectures about algorithmic collusion".

5 Der Beitrag behandelt die wettbewerbsbeschränkenden Vereinbarungen; zur Frage des Missbrauchs einer marktbeherrschenden Stellung durch algorithmenbasiertes Marktverhalten s. den Beitrag von Petra Pohlmann in diesem Band. 
Algorithmenbasierter Handel und Kartellverbot

\section{Fälle}

\section{Die Poster-Fälle}

Mit den Poster-Fällen sind ein US-amerikanischer ${ }^{6}$ und ein britischer Fall ${ }^{7}$ gemeint, in denen es um den Verkauf von Postern, Drucken und gerahmter Kunst über Amazon Marketplaces ging, also denjenigen Teil der AmazonPlattform, über den nicht Amazon selber, sondern Dritte als Verkäufer auftreten. Posterhändler sprachen die Preise für die betreffenden Produkte ab. Insofern lag ein klassisches Preiskartell vor. Der Postermarkt zeichnet sich allerdings durch große Heterogenität, nämlich die Existenz tausender verschiedener Produkte aus. Die individuelle und manuelle Preiskoordination erwies sich deshalb als "a laborious and time-consuming exercise". ${ }^{8}$ Die Anbieter gingen deshalb dazu über, Preissoftware einzusetzen und diese zum Zweck der Preiskoordination anzupassen. ${ }^{9}$

$\mathrm{Zu}$ unterstreichen ist die Tatsache, dass die Abredepartner nicht etwa identische, sondern unterschiedliche Softwareprodukte einsetzten. ${ }^{10}$ Für die Zwecke des Kartellrechts ist es unerheblich, welche Instrumente eingesetzt werden, wenn nur klar ist, dass ihr Einsatz die Festsetzung der Verkaufspreise bezweckt. Aufschlussreich ist auch ein anderes Detail des britischen Posterfalls, das eine Antwort auf die Frage gibt, wie man Preise abspricht, ohne von Kartellaußenseitern unterboten zu werden. Dies geschieht dadurch, dass man zwar im Verhältnis zu Dritten eine wettbewerbsfreundliche Preisunterbietungsregel vorsieht, von dieser aber unter Einsatz einer ignore list Ausnahmen im Verhältnis zu den Kartellpartnern

6 U.S. District Court, Northern District of California, 30.4.2015 - U.S. v. Topkins, No. CR 15-00201.

7 Competition and Markets Authority (CMA), 12.8.2016, Case 50223 - Online sales of posters and frames, abrufbar unter: www.gov.uk/cma-cases/online-sales-of-discretionary-consumer-products.

8 Ibid., N. 3.66.

9 Die Machbarkeit algorithmischer Kartelle wird häufig mit dem Hinweis angezweifelt, dass die wirtschaftliche Realität komplex sei, und dass die Anwesenheit einer Vielzahl von Akteuren für Instabilität sorge. Der Posterfall zeigt, dass man algorithmische Koordination gerade dann benötigen kann, wenn es kompliziert wird. Da manuelle Koordination angesichts der Heterogenität des Produkts nicht praktikabel ist, geht man zu algorithmischer Koordination über. Das Kartell zwischen mittelgroßen Anbietern "gelingt" (s. Fn. 12), obwohl die Marktzutrittsschranken für den Posterverkauf über das Internet denkbar niedrig sind und die Marktform ein Polypol ist.

$10 C M A$ (Fn. 7), N. 3.46, 3.62-3.63, 3.80, 5.17. 
macht. ${ }^{11}$ Dann ist eine Reaktion auf tiefere Preise von Dritten möglich, ohne dass die Preiskoordination der Kartellanten im Innenverhältnis gestört wird. Diese Lösung wird besonders interessant auf Märkten sein, auf denen große Produktdiversität herrscht und Dritte nicht alle Produkte der Kartellteilnehmer führen bzw. nicht überall günstigere Angebote vorlegen. ${ }^{12}$

\section{Einordnung}

Die Posterfälle zeigen, dass Preissoftware den Wettbewerb intensivieren, aber auch beschränken kann. ${ }^{13}$ Offenbar ist es möglich, sogar gängige Preissoftware so anzupassen, dass nicht mehr optimale Preise im Wettbewerb, sondern koordinierte Preise im Kartell das Ziel sind. ${ }^{14}$ Die Fälle zeigen aber auch, dass Algorithmen eine sehr untergeordnete Rolle in der Kartellbildung einnehmen können. In Anlehnung an den Terminus des Computer-aided design (CAD) lässt sich von Computer-aided cartels sprechen. Die einschlägigen Fälle unterscheiden sich damit kaum von "klassischen" Kartellfällen, da Algorithmen nur in dienender Funktion eingesetzt werden, nämlich als Werkzeug benutzt werden, um ein zwischen Menschen verabredetes Kartell umzusetzen. Für die Anwendung des Kartellrechts ergeben sich in einer solchen Konstellation keine besonderen Schwierigkeiten. Es ist deshalb kein Zufall, dass die Posterfälle den Beginn der Diskussion um algorithmische Kartelle markieren.

In ihrem einflussreichen Buch "Virtual Competition"15 haben Ariel Ezrachi und Maurice E. Stucke bereits in einem frühen Zeitpunkt (nämlich 2016) klargestellt, dass dies nur der Ausgangspunkt ist. Die soeben

11 Ibid., N. 3.81 ff., 5.18.

12 S. den Erfolg der Preisabsprachen im britischen Posterfall: "[We] have now activated the Amazon repricing software and it is working perfectly. Trod should be happy with the outcome as we are now the same price as them on $99 \%$ of listings", ibid., N. 3.72.

13 S. die Aussage des (damaligen) CMA-Direktors Stephan Blake zum britischen Posterfall: "Online pricing tools, such as automated repricing software, can also help sellers compete better, for the benefit of consumers. In this case, however, the parties used repricing software to implement an illegal agreement to deny consumers these benefits", abrufbar unter: www.gov.uk/government/news/onlineseller-admits-breaking-competition-law.

14 Zur Funktionsweise von Preisalgorithmen s. Monopolkommission, Wettbewerb 2018 - XXII. Hauptgutachten, 2018, S. 65 f. N. 169 ff.

15 Ezrachi/Stucke (Fn. 3). 
dargestellte Situation, in der Algorithmen lediglich als Werkzeuge zur Kartellimplementierung eingesetzt werden, bezeichnen sie als Messenger. Davon unterscheiden sie drei weiterführende "Collusion Scenarios", nämlich Hub-and-Spoke, Predictable Agent und Artificial Intelligence. ${ }^{16}$ In der Huband-Spoke-Konstellation ("Sternverträge") koordinieren Konkurrenten sich nicht direkt, sondern stehen in Verbindung mit einem Dritten, z.B. einer Plattform oder einem Dienstleister, der dafür sorgt, dass die "Speichen" ihr Verhalten aufeinander abstimmen. Es handelt sich also um eine indirekte Form der Verhaltenskoordination. In der dritten Fallgruppe setzen Unternehmen ohne Kontakt zueinander Preissoftware ein, welche die eigenen Preise selbständig an fremde Preise anpasst. Die Geschäftsleitung ist der Prinzipal, die Software der Agent, das Ergebnis kann tacit collusion sein, deren kartellrechtliche Einordnung Schwierigkeiten bereitet. Am weitesten geht das KI-Szenario: Hier wird die Verbindung zu menschlichem Verhalten getrennt, und die Software erkennt selbständig, dass Kollusion mit der künstlich intelligenten Software anderer Unternehmen die Gewinne steigert.

Bei der Fortsetzung der Fallübersicht wird sich zeigen, dass neben dem Messenger-Szenario bereits heute die Hub-and-Spoke-Konstellation einschlägig ist, während Predictable Agents und Künstliche Intelligenz im Kartellzusammenhang noch Zukunftsmusik sind, jedenfalls noch kein einschlägiger Kartellrechtsverstoß durch Behörden oder Gerichte festgestellt worden ist.

\section{Fahrdienstvermittler}

a) Uber

In der Sharing Economy erfolgt die Preisbildung häufig dynamisch; Algorithmen bestimmen kontinuierlich, welcher Preis gerade gilt. ${ }^{17}$ Ein Beispiel ist der Fahrdienstleister Uber: Beim Uber Surge Pricing fließen in Echtzeit das jeweilige Angebot und die jeweilige Nachfrage (einschließlich ihrer Schwankungen wegen Hauptverkehrszeit, schlechten Wetters oder besonderer Vorkommnisse) in die Preisbildung ein. Vor allem aber sind

16 Ezrachi/Stucke (Fn. 3), S. 35 ff.

$17 \mathrm{Zu}$ kartellrechtlichen Problemen der Sharing Economy s. Safron, The Application of EU Competition Law to the Sharing Economy, Stanford-Vienna European Union Law Working Paper No. 27, Stanford, Vienna 2018, abrufbar unter: wwwcdn.law.stanford.edu/wp-content/uploads/2018/02/safron_eulawwp27.pdf. 
Uber-Fahrer in der Preisbildung nicht frei. Sie benutzen die Uber-App, die ihnen den jeweiligen Preis vorgibt. Verhandlungen mit den Kunden sind traditionell nicht möglich. ${ }^{18}$ Es stellt sich die Frage, ob dies ein horizontales Preiskartell in Hub-and-Spoke-Form zwischen den Uber-Fahrern vermittelt durch das Unternehmen Uber darstellt. Eine Voraussetzung hierfür wäre, dass die Uber-Fahrer selbständige Unternehmer sind, zwischen denen durch die Uber-App der Preiswettbewerb ausgeschaltet würde. Ein UberKunde hat 2015 eine class action mit diesem Vorwurf initiiert und beim Bundesbezirksgericht für das südliche New York eingereicht. Nachdem zunächst die Tragweite einer Schiedsabrede zu klären war, verwies das Bezirksgericht den Fall an einen Schiedsrichter. Dieser hat im Februar 2020 die Klage abgewiesen. Das Bezirksgericht wies eine hiergegen gerichtete Motion to Vacate ab. ${ }^{19}$ Da mit der gerichtlichen Überprüfung von Schiedssprüchen nur bestimmte Exzesse (in casu Voreingenommenheit) überprüft werden können, und der Schiedsspruch selbst, soweit ersichtlich, nicht veröffentlicht ist, bleibt offen, aus welchen Gründen die Gruppenklage abgewiesen worden ist. Uber jedenfalls hat den Vorwurf der Preisabsprache stets zurückgewiesen. ${ }^{20}$

\section{b) Webtaxi Luxemburg}

Aufschlussreich ist die Webtaxi-Entscheidung der luxemburgischen Kartellbehörde. Hier ging es um eine ganz ähnliche Fahrdienstleistungs-App wie bei Uber: Ein zentral gesteuertes System bestimmt auf Anfrage das kun-

18 Anders aber das im Juli 2020 als weltweite Premiere in der Schweiz eingeführte System: Die Fahrer können den von Uber vorgeschlagenen Preis individuell anpassen, s. NZZ v. 2.9.2020, S. 11.

19 US District Court, Southern District of New York, 3.8.2020 - Spencer Meyer v. Travis Kalanick and Uber Technologies, No. 15 Civ. 9796, mit Nachweis der anderen Entscheidungen in diesem Verfahren. Der Schiedsrichter hatte in seinen Schlussbemerkungen die offenbar humorvoll gemeinte Aussage gemacht, dass er aus Angst um seine Sicherheit zugunsten von Uber entschieden habe. Richter Jed S. Rakoff vom District Court bemerkte hierzu: "While perhaps inappropriate (or, worse yet, not as humorous as some of the arbitrator's better jokes), the remarks are not inconsistent with impartiality once their patently jestful intent is recognized", ibid., S. 8.

20 Uber-Gründer Travis Kalanick: "We are not setting the price. The market is setting the price. We have algorithms to determine what that market is", zitiert von Priluck, When Bots Collude, The New Yorker v. 25.4.2015, abrufbar unter: www.newyorker.com/business/currency/when-bots-collude. 
dennächste Taxi und berechnet den Preis dynamisch nach der aktuellen Lage von Angebot und Nachfrage. Das System gibt den angeschlossenen Fahrern den Preis vor; individuelle Preisverhandlungen mit den Kunden sind nicht möglich. Im Unterschied zu Uber sind an die luxemburgische App nicht nur eigene Taxis des Plattformunternehmens, sondern auch andere Taxiunternehmer angeschlossen, die zweifelsfrei selbständig sind, so dass an der Anwendbarkeit des (luxemburgischen) Kartellverbots kein Zweifel bestand.

Die luxemburgische Kartellbehörde entschied, dass zwar eine horizontale Preisabsprache in Form einer "politique tarifaire unifiée" vorliege, die aber aus Gründen der wirtschaftlichen Effizienz gerechtfertigt sei. Die zentrale Vermittlungsplattform verkürze Wartezeiten und vermeide Leerfahrten. Hierdurch würden Emissionen reduziert, was ebenfalls als Effizienzvorteil zu werten sei. Die Wettbewerbsbeschränkung sei zur Realisierung der Effizienzgewinne auch erforderlich: Ließe man Preisverhandlungen mit den Kunden zu, fiele die Wahl nicht mehr unbedingt auf den geographisch nächsten Fahrer, so dass es nicht zu denselben Einsparungen käme. ${ }^{21}$

Die Entscheidung ist für die Beurteilung von Koordinationsaktivitäten in der digitalen Ökonomie von größter Bedeutung. Schon vorher stand aufgrund des Cartes Bancaires-Urteils des Europäischen Gerichtshofs fest, dass Kernbeschränkungen, wie z.B. horizontale Preisbeschränkungen, bei Plattformkomplexität oder indirekten Netzwerkeffekten der Kategorie der bezweckten Wettbewerbsbeschränkung entzogen sein können. ${ }^{22}$ Die Entscheidung der luxemburgischen Kartellbehörde veranschaulicht, dass auch eine Effizienzrechtfertigung nach Art. 101 Abs. 3 AEUV greifen kann. ${ }^{23}$ Der oft gehörte Satz, dass eine Rechtfertigung von Kernbeschränkungen zwar theoretisch möglich, praktisch aber nur völlig vereinzelt in Betracht käme, wird angesichts der gewaltigen Effizienzsteigerungen in der digitalen Ökonomie durch Wiederholung nicht richtiger. Das europäische Kartellrecht sieht keine per se-Verbote vor. Gerade auch im Hinblick auf das

21 Conseil de la concurrence (Luxembourg), 7.6.2018 - Webtax, abrufbar unter: https:// concurrence.public.lu/fr/decisions/ententes/2018/decision-2018-fo-01.html.

$22 \mathrm{EuGH}, 11.9 .2014$, Rs. C-67/13 P - Groupement des cartes bancaires/Kommission, ECLI:EU:C:2014:2204, Rn. $48 \mathrm{ff}$.

23 Zur Effizienzrechtfertigung bei algorithmischer Preisbestimmung (auch im Hinblick auf Uber) s. Göhsl, Algorithm Pricing and Article 101 TFEU - Can Competition Law deal with algorithm pricing?, WuW 2018, 121 (123). 
Thema der algorithmischen Koordination sind die Voraussetzungen für eine Effizienzrechtfertigung sorgsam zu prüfen. ${ }^{24}$

\section{Eturas}

Technisch weit weniger komplex als ein System dynamischer Preisbildung ist der Sachverhalt, welcher der Eturas-Entscheidung des Europäischen Gerichtshofs ${ }^{25}$ zugrunde liegt. In Litauen benutzten ca. 30 Reisebüros das von dem Unternehmen Eturas entwickelte Online-Buchungssystem E-TURAS. $\mathrm{Zu}$ einem bestimmten Stichtag griff Eturas in den Wettbewerbsparameter Preis ein: Das Buchungssystem begrenzte die Höhe der Rabatte auf 3 Prozent. Zwar war es technisch möglich, diese Begrenzung zu umgehen. Wegen des hiermit verbundenen Aufwands kam es aber dazu, dass die meisten Reisebüros, die vorher großzügigere Rabatte gewährt hatten, diese nun auf 3 Prozent begrenzten. Eturas informierte die angeschlossenen Reisebüros nicht per E-Mail, sondern über eine Systemmitteilung innerhalb des Buchungssystems E-TURAS, die in das passwortgeschützte Feld "Informationsmitteilungen" eingestellt wurde. Diese Mitteilung enthielt u.a. folgenden Satz: "Für Reisebüros, die einen Rabatt von mehr als $3 \%$ angeboten haben, wird [dieser] ab $14 \mathrm{Uhr}$ automatisch auf $3 \%$ reduziert."26 Die litauische Kartellbehörde sah in dieser Vorgehensweise eine wettbewerbsbeschränkende abgestimmte Verhaltensweise und verhängte Geldbußen, wogegen sich die Reisebüros vor Gericht wehrten. Sie brachten vor, dass es sich bei der Systemmitteilung um eine einseitige Handlung von Eturas handele. Sie hätten diese Mitteilung nicht erhalten, jedenfalls nicht gelesen, also nicht gebilligt, womit es an einer abgestimmten Verhaltensweise fehle.

Der im Wege des Vorabentscheidungsverfahrens befasste Europäische Gerichtshof stellt das Merkmal der Kenntnis in den Mittelpunkt. Mit Kenntniserlangung greife die tatsächliche Vermutung, dass das betreffende Unternehmen sich an einer abgestimmten Verhaltensweise beteiligt habe. ${ }^{27}$ Es verstoße gegen die Unschuldsvermutung, aus dem bloßen

24 S. hierzu Monopolkommission (Fn. 14), S. 85 f. N. 259 f.; Salaschek/Serafimova, Preissetzungsalgorithmen im Lichte von Art. 101 AEUV - Innovation des Preiswettbewerbs oder kartellrechtliche Grauzone?, WuW 2018, 8 (12).

25 EuGH, 21.1.2016, Rs. C-74/14 - Eturas, ECLI:EU:C:2016:42.

26 S. EuGH - Eturas (Fn. 25), Rn. 10.

27 EuGH - Eturas (Fn. 25), Rn. 44; zur Umstoßung dieser Vermutung s. N. 46. 
Versenden der Systemmitteilung auf Kennen (oder Kennenmüssen ${ }^{28}$ ) zu schließen. ${ }^{29}$

Im Hinblick auf den vorliegenden Zusammenhang sind zwei Aspekte des Eturas-Urteils hervorzuheben. Erstens liegt im Sinne der Ezrachi/Stucke'schen Klassifikation ${ }^{30}$ eine Hub-and-Spoke-Konstellation vor: Die Reisebüros koordinieren den Maximalrabatt nicht direkt miteinander, sondern diese Aufgabe wird von einem Dritten, nämlich dem Online-Buchungssystem E-TURAS übernommen. ${ }^{31}$ Und zweitens liegen keine fortgeschrittenen Algorithmen zugrunde (nicht einmal solche über dynamische Preisbildung), sondern das Buchungssystem nimmt eine simple Rabattbeschränkung auf 3 Prozent vor. Für einen solchen Fall erscheint das vom Europäischen Gerichtshof herangezogene Kriterium der Kenntnis angemessen. Es stellt sich aber die Anschlussfrage, ob dieses Kriterium auch dann noch angemessen ist, wenn die Algorithmen so komplex sind, dass die Fixierung von Wettbewerbsparametern mehr und mehr den Menschen entzogen und an Maschinen delegiert wird. Die Frage der angemessenen kartellrechtlichen Beurteilung algorithmischer Koordination soll im Folgenden beurteilt werden.

28 Bedauerlicherweise schafft der Gerichtshof Unklarheit, indem er an manchen Stellen des Urteils dem Kennen das Kennenmüssen gleichstellt, an anderen hingegen nicht; s. die Analyse bei Heinemann/Gebicka, Can Computers form Cartels?, 7 Journal of European Competition Law \& Practice 431, 436 (2016). Für eine Willensübereinstimmung ist Vorsatz erforderlich; Fahrlässigkeit reicht nicht aus, s. Pohlmann, Algorithmen als Kartellverstöße, in: Kokott/Pohlmann/Polley (Hrsg.), FS Dirk Schroeder, 2018, S. 633 (645).

29 EuGH -Eturas (Fn. 25), Rn. 39.

30 S.o. II.2.

31 Gegen eine Klassifizierung des Eturas-Falls als Hub-and-Spoke-Kollusion allerdings Generalanwalt Maciej Szpunar: Für ihn beschränkt sich diese Konstellation auf Fälle, in denen die vertikale Kommunikation auch als zulässiger geschäftlicher Dialog gedeutet werden könnte, während im Eturas-Fall von vornherein klar sei, dass die Rabattbegrenzung unzulässig sei, s. Szpunar, 16.7.2015, Rs. C-74/14 - Eturas, ECLI:EU:C:2015:493, Rn.65. Es leuchtet allerdings nicht ein, warum man den Begriff der Hub-and-Spoke-Kollusion so restriktiv auslegen soll. Entscheidend ist die Tatsache, dass die beschränkende Abrede nicht direkt zwischen den Marktteilnehmern, sondern durch Interaktion mit einem Dritten erfolgt. In diesem Sinn auch Safron (Fn. 17), S. 32. 


\section{Algorithmische Koordination}

\section{Preissoftware}

Der Einsatz von Preissoftware im Onlinehandel hat stark steigende Tendenz. Bereits im Abschlussbericht über die Sektoruntersuchung zum elektronischen Handel aus dem Jahr 2017 stellte die Europäische Kommission fest, dass zwei Drittel derjenigen Einzelhändler, welche die Online-Preise von Wettbewerbern verfolgen, eine solche Software nutzen. ${ }^{32}$ Preissoftware ist imstande, die Preisentwicklung im Markt zu beobachten (price tracking), Preisänderungen anderer Marktteilnehmer zeitnah zu überwachen (price monitoring) und die eigenen Preise hieran anzupassen (price setting). Die Algorithmen profitieren von der wachsenden Verfügbarkeit grosser Datenbestände (Big Data) und den sich ständig verbessernden Möglichkeiten, diese auszuwerten (Big Analytics). ${ }^{33}$

Mittlerweile liegen die ersten Studien zu Wirkungsweise und Potential der Preissoftware aus kartellrechtlicher Sicht vor. So hat die britische Kartellbehörde 2018 ein Arbeitspapier über den Einsatz von Algorithmen im Hinblick auf Kollusion und personalisierte Preise verfasst. ${ }^{34}$ Es schafft nicht nur eine theoretische Basis, sondern nimmt auch eine erste Untersuchung existierender Preissoftware vor. So ist es beim Verkauf über Amazon Marketplace beispielsweise möglich, Preisregeln der Plattform zu aktivieren, z.B. die Match Low Price-Regel, mit der automatisch der niedrigste von Konkurrenten angewandte Preis übernommen werden kann. Es können aber auch kompliziertere Funktionen von Drittanbietern eingesetzt werden. Schließlich ist es möglich, die Preissetzung ganz an Dritte zu delegieren. Auch wenn offenbar die Personalisierung von Preisen noch nicht sehr verbreitet ist, kommt es häufiger zu Preisänderungen als in der Vergangenheit. Einzelne Verkäufer ändern ihre Preise mehrere hundert Male am Tag. ${ }^{35}$

32 Europäische Kommission, Abschlussbericht über die Sektoruntersuchung zum elektronischen Handel, COM(2017) 229 final, Brüssel, 10.5.2017, N. 13.

33 S. hierzu Burri, Understanding the Implications of Big Data and Big Data Analytics for Competition Law, in: Mathis/Tor (Hrsg.), New Developments in Competition Law and Economics, 2019, S. $241 \mathrm{ff}$.

$34 C M A$, Pricing Algorithms - Economic working paper on the use of algorithms to facilitate collusion and personalised pricing, 2018, abrufbar unter: www.gov.uk/ government/news/algorithm-research-builds-on-work-in-digital-markets.

35 Ibid., N. 3.5, $7.7 \mathrm{ff}$. 
Auch die deutsche und die französische Kartellbehörde haben sich des Algorithmenthemas angenommen und 2019 eine gemeinsame Studie veröffentlicht. ${ }^{36}$ Auch diese Studie legt eine Typologie der verschiedenen Algorithmen vor und unterscheidet verschiedene Szenarien, in denen Algorithmen in wettbewerbsrelevanter Weise zum Einsatz kommen können. In ökonomischer Hinsicht wird die Plausibilität von Kollusion untersucht. Schließlich arbeitet die Studie die praktischen Probleme heraus, denen Kartellbehörden bei der Untersuchung algorithmischer Kollusion begegnen. Eine wichtige Unterscheidung, die in der Studie getroffen wird, ist der Unterschied zwischen Koordination auf der Ebene der Algorithmen (code level) und auf der Ebene der Inputfaktoren (data level). Zu einer relevanten Koordination auf code level kommt es beispielsweise dann, wenn Wettbewerber gleiche oder ähnliche Algorithmen einsetzen oder die Preissetzung ganz auf einen Dritten delegieren. Koordination auf data level liegt z.B. vor, wenn Algorithmen zum Datenaustausch verwendet werden, oder Wettbewerber denselben Datenpool nutzen. ${ }^{37}$ Auf Fragen des Datenaustauschs sind die allgemeinen kartellrechtlichen Regeln über den Informationsaustausch anwendbar. ${ }^{38}$ Die Privilegierung des Austauschs alter, öffentlich bekannter und aggregierter Daten sei zu überdenken, wenn diese zum Training des Algorithmus mit dem Effekt der Preiskoordination verwendet werden. ${ }^{39}$

Diese und andere ${ }^{40}$ Untersuchungen sind von größtem Wert für die Frage, wie die Algorithmen im Hinblick auf das Wettbewerbsthema funk-

36 Autorité de la concurrence / Bundeskartellamt, Algorithms and Competition, 2019, abrufbar unter: www.bundeskartellamt.de/SharedDocs/Meldung/EN/Meldungen \%20News\%20Karussell/2019/06_11_2019_algorithms\%20and\%20competition.ht $\mathrm{ml}$.

37 Autorité de la concurrence / Bundeskartellamt (Fn. 36), S. 33 ff.

38 S. bereits Bundeskartellamt, 25.9.2000 - Covisint, K\&R 2000, 604: Ein Gemeinschaftsunternehmen großer Automobilhersteller sollte die Zulieferung von Vorprodukten über eine eigens diesem Zweck dienende Internetplattform modernisieren. Die Rechtmäßigkeit wurde davon abhängig gemacht, dass Marktteilnehmer keinen Einblick in die Konditionen der über die Plattform abgeschlossenen Geschäfte erhalten. Näher zu den Vorgaben für Informationsaustausch im Kontext der Algorithmen s. Heinemann, Algorithmen als Anlass für einen neuen Absprachebegriff?, SZW/RSDA 2019, S. 18 (26f.).

39 Autorité de la concurrence / Bundeskartellamt (Fn. 36), S. 33 f., 39.

40 S. beispielsweise das Kapitel "Algorithmen und Kollusion" der Monopolkommission (Fn. 14), S. 62 - 88, sowie die Studie der portugiesischen Kartellbehörde: Autoridade da Concorrência, Digital Ecosystems, Big Data and Algorithms, 2019, abrufbar unter: www.concorrencia.pt/vEN/Estudos_e_Publicacoes/Estudos_Economic os/Other/Pages/Digital-Ecosystems-Executive-Summary_.aspx. 
tionieren, welches Potential sie haben und wie die Möglichkeiten algorithmischer Kollusion in ökonomischer Hinsicht zu fassen sind. Allerdings ist das Wissen um die Wirkungsweise von Preissoftware ausbaufähig. Wünschenswert wäre eine noch praktischere Herangehensweise, nämlich eine Untersuchung existierender Preissoftware im Hinblick auf ihr kollusives Potential, und das aus Sicht und mit der Erfahrung konkreter Marktteilnehmer. ${ }^{41}$ Wahrscheinlich wird es kartellbehördlicher Einzelfalluntersuchungen bedürfen, um exemplarisch zu erfahren, welche wettbewerbsbeschränkenden Möglichkeiten Preissoftware eröffnet.

Um Missverständnisse zu vermeiden: Die vorstehenden Ausführungen beziehen sich auf die Risiken, die dem Wettbewerb durch den Einsatz von Preissoftware drohen. Demgegenüber steht die wettbewerbsfördernde Wirkung: Preissoftware wird in allererster Linie dazu eingesetzt, um den Absatz zu optimieren und den Gewinn zu maximieren, was in der Regel dazu führen wird, dass man versucht, die Preise der Konkurrenz zu unterbieten oder zumindest auf konkurrenzfähigem Niveau zu halten. Wie so häufig in digitalen Zusammenhängen lässt sich Ambivalenz beobachten. Die digitalen Errungenschaften werden in erster Linie zur Belebung des Wettbewerbs führen. Es sind aber auch wettbewerbsbeschränkende Einsatzmöglichkeiten denkbar. Das gilt auch für den Einsatz von Preissoftware.

Um wiederum ein Beispiel aus der kartellbehördlichen Praxis aufzugreifen: Infolge der bereits erwähnten Sektoruntersuchung zum elektronischen Handel hat die Europäische Kommission konkrete Fälle untersucht. Nach ihren Feststellungen haben vier Elektronikhersteller die Freiheit ihrer Onlinehändler bei der Festsetzung ihrer Wiederverkaufspreise eingeschränkt. Wenn die Hersteller feststellten, dass einer der Händler mit den Preisen zu weit nach unten ging, erfolgte eine Kontaktaufnahme, bei der die Einhaltung der empfohlenen Weiterverkaufspreise angemahnt und mit Lieferstopp gedroht wurde. Die Europäische Kommission stellte verbotene vertikale Preisbindung fest und verhängte hohe Geldbußen..$^{42}$ Für den vorliegenden Zusammenhang sind zwei Aspekte des Falls erhellend: Einerseits setzten die Hersteller Preissoftware ein, um die Preisentwicklung im Onlinehandel zu verfolgen. Dies führte zu weitaus rascherem Eingreifen, als es bei traditioneller Preisbeobachtung möglich gewesen wäre. ${ }^{43}$ Ande-

41 Ansätze hierzu finden sich in Autoridade da Concorrência (Fn. 40), N. 208 ff.

42 Europäische Kommission, 24.7.2018 - Asus, Denon \& Marantz, Philips und Pioneer, ABl. 2018 C 338/13 u.a.

43 Europäische Kommission, 24.7.2018 - Elektronischer Handel, Pressemitteilung IP/18/4601: "Ferner konnten die Hersteller durch hochentwickelte Überwa- 
rerseits hatte die Preisbindung einen digital vermittelten Einfluss weit über die betroffenen Händler hinaus: Auch die nicht im Preis gebundenen Händler setzen Preissoftware ein, die auf die durch die Preisbindung bewirkte Anhebung des Preisniveaus reagiert und damit in Form eines vertikalen umbrella-Effekts ${ }^{44}$ zu höheren Preisen im Gesamtmarkt führt. ${ }^{45}$

\section{Stillschweigende Kollusion}

Wird Preissoftware eingesetzt, um die Wettbewerber zu unterbieten, liegt offenbar keine Koordination vor; im Gegenteil, der Wettbewerb wird hierdurch belebt. Problematisch wird es, wenn Algorithmen eingesetzt werden, um in die Preise von Wettbewerbern einzusteigen, z.B. Preiserhöhungen eines Wettbewerbers nachzuvollziehen. Eine klar wettbewerbsbeschränkende Vereinbarung liegt vor, wenn die Unternehmen die Verwendung derselben oder funktionsgleicher Software verabredet haben oder ihre Software koordiniert programmieren. Ausreichend für eine tatbestandsmässige Vereinbarung ist die Verwendung koordinierender Elemente, z.B. die Vereinbarung einer Ausnahme von Preisunterbietungsregeln zugunsten einzelner Konkurrenten unter Verwendung einer ignore-Liste. ${ }^{46}$

Wie aber verhält es sich, wenn kein Kontakt zwischen den Konkurrenten besteht und die Preissoftware autonom eingesetzt wird? Folgendes Beispiel sei gegeben: Ein Marktführer testet Preiserhöhungen. ${ }^{47}$ Er stellt fest, dass die Wettbewerber dank algorithmenbasierter repricing tools ihre Preise jeweils unmittelbar nach oben anpassen. Das früher bestehende

chungsinstrumente die Wiederverkaufspreisbildung im Vertriebsnetz verfolgen und im Falle von Preissenkungen rasch eingreifen".

44 Der umbrella-Effekt wurde im Hinblick auf (horizontale) Kartelle entwickelt und beschreibt das Phänomen, dass Kartelle nicht nur die Preise der Kartellunternehmen, sondern auch die Preise nicht kartellbeteiligter Konkurrenten in die Höhe treiben. Der E-Commerce-Fall der Europäischen Kommission zeigt, dass ein solcher umbrella-Effekt auch die Folge vertikaler Wettbewerbsbeschränkungen sein kann.

45 Europäische Kommission (Fn. 43): "Viele Online-Einzelhändler, auch die größten, setzen Preisalgorithmen ein, durch die ihre Einzelhandelspreise automatisch an die Preise der Wettbewerber angepasst werden. Daher wirkten sich die Beschränkungen für die Online-Einzelhändler des Niedrigpreissegments auf die gesamten Online-Preise für die jeweiligen Elektronikprodukte aus".

46 S.o. bei Fn. 11.

47 Zum Signalling im algorithmischen Kontext s. Monopolkommission (Fn. 14), S. 69 N. $187 \mathrm{f}$. 
Risiko, beim Ausprobieren einer Preiserhöhung Kunden zu verlieren, besteht nicht mehr, da die Konkurrenz mit ihren Preiserhöhungen nicht auf sich warten lässt. Es ist vorgeschlagen worden, solche Fälle unter den traditionellen Absprachebegriff zu subsumieren. Programmiert ein Unternehmen seine Preissoftware so, dass Preiserhöhungen des Marktführers jeweils nachvollzogen werden, stelle dies ein Angebot zum Abschluss einer wettbewerbsbeschränkenden Vereinbarung an den Marktführer dar, das dieser durch eine Preiserhöhung annehme. ${ }^{48}$ In eine ähnliche Richtung zielt der Vorschlag, gegenseitige Informationssignale dann unter den Abredebegriff zu fassen, wenn sie zu einem suprakompetitiven Gleichgewicht führen und die Konsumentenwohlfahrt vermindern. Eine Wirkungsanalyse sei deshalb schon bei der Feststellung einer abgestimmten Verhaltensweise erforderlich..$^{49}$ Auf eine Erweiterung des Begriffs der abgestimmten Verhaltensweise zielt auch folgender Vorschlag: Werden Preise in einer Geschwindigkeit aneinander angeglichen, welche die Schiedsrichterrolle der Verbraucher ausschließt, liege nicht mehr bloßes Parallelverhalten, sondern Koordinierung vor. ${ }^{50}$ Schließlich wird die Einbeziehung von "Plus-Faktoren" diskutiert: Kommt es auf Märkten zu suprakompetitiven Gleichgewichten und liegt ein solcher Faktor vor, wird eine tatbestandsmäßige Koordinierung angenommen. Als Plus-Faktoren werden dynamische Preissetzung, die gemeinsame Nutzung von Datenbanken Dritter oder der Einsatz selbstlernender Preisalgorithmen vorgeschlagen. ${ }^{51}$

Die Vorschläge eint das Bestreben, den Abredebegriff, der bisher auf anthropozentrische Kriterien wie Willensübereinstimmung, meeting of the minds oder Kenntnis abstellt, an die Herausforderungen maschineller Kontexte anzupassen. Wie wendet man die traditionellen Kategorien auf Computersysteme an, die immer weniger menschliches Einschreiten erfordern, und durch die zwischenmenschliche immer mehr durch intermaschinelle Kommunikation ersetzt wird? Hier besteht sicherlich Handlungsbedarf, der aber auf zwei Vorgaben reagieren muss: Erstens müssen die vorgeschlagenen Kriterien praktikabel sein, und zweitens dürfen sie nicht zu

48 Capobianco/Gonzaga, Algorithms and Competition: Friends or Foes?, Competition Policy International 2017, S. 4, abrufbar unter: www.competitionpolicyinternational.com/algorithms-and-competition-friends-or-foes.

49 Thomas, Harmful Signals: Cartel Prohibition and Oligopoly Theory in the Age of Machine Learning, 15 Journal of Competition Law \& Economics 159 (2019).

50 Wolf, Algorithmengestützte Preissetzung im Online-Einzelhandel als abgestimmte Verhaltensweise, NZKart 2019, S. 2 (9 f.).

51 Capobianco/Gonzaga (Fn. 48), S. 4; Gal/Elkin-Koren, Algorithmic Consumers, 30 Harvard Journal of Law \& Technology 309, $346 \mathrm{f}$. (2017). 
weit gehen, also nicht wettbewerbsfördernde Verhaltensweisen erfassen. Die bisher gemachten Vorschläge sind entweder schwierig in der praktischen Handhabung, oder sie sind over-inclusive. So ist dem Vorschlag von Plus-Faktoren eine gewisse Innovationsfeindlichkeit inhärent: Wenn beispielsweise der Einsatz dynamischer Preisbildung Probleme mit den Kartellbehörden heraufbeschwört, werden Anreize gesetzt, hierauf lieber zu verzichten. Das ist den dynamischen Funktionen des Wettbewerbs abträglich. ${ }^{52}$

Am radikalsten ist der Vorschlag, auf besondere Konstruktionen zu verzichten und tacit collusion generell als tatbestandsmäßige Koordination anzuerkennen. Dieser für das US-amerikanische Antitrust-Recht gemachte Vorschlag argumentiert, dass das wirtschaftliche Ergebnis klassischer und stillschweigender Koordination dasselbe sei, nämlich eine gleichförmige Setzung von Preisen erreicht werde.$^{53}$ Deshalb solle tacit collusion wie eine Wettbewerbsabrede behandelt werden. ${ }^{54}$ De lege lata ist im europäischen Kartellrecht eine solche Ausdehnung nicht möglich. ${ }^{55}$ Aber auch rechtspolitisch sollte dem Vorschlag nicht gefolgt werden. ${ }^{56}$ Man würde dann nämlich auf das Erfordernis einer besonderen Kommunikation oder eines Kontakts zwischen den Unternehmen verzichten und an reine Marktentwicklungen und Marktergebnisse anknüpfen. Es wäre verboten, dieselben Preise wie die Wettbewerber zu verlangen oder sogar, die Preise in dieselbe Richtung wie diese zu bewegen. Man wäre verpflichtet, andere Preise als die Wettbewerber zu verlangen, um dem Kollusionsvorwurf zu entgehen.

52 Skeptisch, jeden koordinationserleichternden Algorithmus als Plus-Faktor zu behandeln, auch Gal, Algorithms as Illegal Agreements, 34 Berkeley Technology Law Journal 67, 116 (2019).

53 S. auch Monopolkommission (Fn. 14), S. 79 N. 230: "Damit ist es denkbar, dass Algorithmen zukünftig eingesetzt werden, um die Wirkung eines Kartells unter Verzicht auf wettbewerbsbeschränkende Vereinbarungen bzw. Verhaltensabstimmungen zu erzielen". Überblick über die Mechanismen, durch die Algorithmen implizite Kollusion erleichtern Picht/Freund, Wettbewerbsrecht auf algorithmischen Märkten, sic! 2018, S. 666 (670).

54 Kaplow, Competition Policy and Price Fixing, 2013. Einen entsprechenden Vorschlag hatte ursprünglich Richard A. Posner gemacht, der sich nun aber davon distanziert hat, s. Posner, Review of Kaplow, Competition Policy and Price Fixing, 79 Antitrust Law Journal 761 (2014).

55 Zimmer, Algorithmen, Kartellrecht und Regulierung, in: Kokott/Pohlmann/ Polley (Hrsg.), FS Dirk Schroeder, 2018, S. 999 (1002); Vorschläge de lege ferenda im Sinn einer Erweiterung auf stillschweigende Kollusion liegen vor, s. z.B. Käseberg/von Kalben, Herausforderungen der Künstlichen Intelligenz für die Wettbewerbspolitik - Preisbildung durch Algorithmen, WuW 2018, S. 2 (5).

56 Skeptisch auch Pohlmann (Fn. 28), S. 653. 
Der Algorithmus dürfte nicht so programmiert werden, dass auf eine Preiserhöhung durch die Konkurrenz mit der Erhöhung der eigenen Preise zu reagieren ist. Auch wenn die Ergebnisse stillschweigender Kollusion unerwünscht sind, würde ein solcher Eingriff zu einer unangemessenen Einschränkung der Preissetzungsfreiheit führen. ${ }^{57}$

\section{Künstliche Intelligenz}

Der höchste Schwierigkeitsgrad wird bei der Frage der Wettbewerbsbeschränkungen durch Künstliche Intelligenz erreicht. Wie in der Einleitung skizziert, herrscht bereits Uneinigkeit in der Frage, wie realistisch ein Szenario ist, in dem selbst-lernende Algorithmen ohne menschliche Intervention zum Ergebnis gelangen, dass Kollusion mit den Algorithmen konkurrierender Unternehmen den Gewinn maximiert. Zwar liegen dem oben referierten Fallrecht noch keine KI-Konstellationen zugrunde. Aber unter Laborbedingungen haben neuronale Computernetze bereits bei Spielen vom Typus "Gefangenendilemma" die Vorteile von Kollusion nicht nur erkannt, sondern auch praktiziert. ${ }^{58}$ Dasselbe gilt für algorithmische Preissetzung. ${ }^{59}$ Allerdings bleibt die Frage offen, ob und wenn ja, in welchem Ausmaß Experimente unter Laborbedingungen und strengen Grundannahmen ${ }^{60}$ auf die wirtschaftliche Realität übertragen werden können. ${ }^{61}$

Auch wenn das wettbewerbsbeschränkende Potential selbst-lernender Algorithmen angesichts des eindrucksvollen und stetig ansteigenden Leistungsausweises der Künstlichen Intelligenz sehr groß erscheint, ist die Forderung der Skeptiker nach einem genaueren Studium der Wirkungswei-

57 Der Zwiespalt zwischen Schädlichkeit stillschweigender Kollusion und Abwesenheit eines überzeugenden Gegenmittels wird deutlich in: U.S. Court of Appeals for the First Circuit, 30.6.1988 - Clamp-all Corporation v. Cast Iron Soil Pipe Institute, et al., 851 F.2d 478, 484: "That is not because such pricing is desirable (it is not), but because it is close to impossible to devise a judicially enforceable remedy for "interdependent" pricing. How does one order a firm to set its prices without regard to the likely reactions of its competitors?".

58 S. Capobianco/Gonzaga (Fn. 48), S. 3.

59 Calvano/Calzolari/Denicolò/Pastorello (Fn. 3), S. $31 \mathrm{f}$.

60 Z.B. Beschränkung auf zwei Akteure, Ausschluss von Marktzutritten, perfekte und symmetrische Information.

61 Autorité de la concurrence / Bundeskartellamt (Fn. 36), S. 50 ff.; zum Unterschied zwischen klar geregelten Brettspielen und der Komplexität der wirtschaftlichen Realität s. Hennes/Schwalbe, Kartellbildung durch lernende Algorithmen?, FAZ v. 13.7.2018, S. 18 . 
se solcher Algorithmen unter Einbeziehung computerwissenschaftlicher und ökonomischer Forschung ${ }^{62}$ nicht nur berechtigt, sondern geradezu selbstverständlich. Auch die mittlerweile vorliegenden Studien ${ }^{63}$ stellen nur einen Anfang dar. Hilfreich in diesem Zusammenhang ist die Checkliste der deutsch-französischen Studie zu möglichen Beweismitteln und Methoden der Informationsgewinnung im Algorithmenzusammenhang. ${ }^{64}$ Ihr liegt das Vorsichtsprinzip zugrunde: Auch wenn nicht klar ist, ob es zu $\mathrm{KI}-$ Kartellen kommen wird, ist es doch besser, vorbereitet zu sein. ${ }^{65}$

So empfehlen die beiden Kartellbehörden beispielsweise, die Gründe, den Ursprung und die Geschäftszwecke für den Einsatz des Algorithmus, den Zeitpunkt der ersten Aufschaltung und die Personen zu ermitteln, die in der Vergangenheit und aktuell für den Algorithmus verantwortlich waren und sind. Ebenso wichtig sind Informationen über Änderungen des Algorithmus im Zeitablauf und die Gründe hierfür. Entsprechend der Unterscheidung zwischen code level und data level ${ }^{66}$ sind auch die Datensätze zu identifizieren, die vom Algorithmus benutzt wurden. Hierbei ist von besonderem Interesse, worauf sich die Daten beziehen, beispielsweise ob es eher um eigene Daten des Unternehmens geht, oder ob auch Daten über Preise von Konkurrenten einfließen. Außerdem ist die Funktionsweise des Algorithmus von großer Bedeutung, z.B. Art der Reaktion auf Preisänderungen durch Konkurrenten, Ähnlichkeit mit den Algorithmen der Wettbewerber und Kommunikation zwischen Algorithmen verschiedener

62 Vgl. z.B. Ulrich Schwalbe, Algorithmen, maschinelles Lernen und kollusives Verhalten, in: Kokott/Pohlmann/Polley (Hrsg.), FS Dirk Schroeder, 2018, S. 739 (740): "preissetzende Algorithmen [werden] in vielen juristischen Beiträgen wie eine mysteriöse Black Box behandelt - es wird nicht erklärt, wie diese Algorithmen funktionieren, was maschinelles Lernen ist, welche Arten von maschinellem Lernen in preissetzenden Algorithmen eingesetzt werden, wie Algorithmen lernen und was sie lernen können".

63 S.o. III.1.

64 Autorité de la concurrence / Bundeskartellamt (Fn. 36), S. 62 ff.

65 Ibid., S. 61: "So far, there are only a few cases involving the analysis of the inner workings of algorithms and there is no indication yet as to which types of cases the competition authorities will face in the future. Accordingly, it is not yet possible to predict whether there is a need for competition authorities to adapt their toolkit, and if so, how. The following overview is thus mostly anticipatory and does not prejudge how competition authorities would investigate future cases". Auch diejenigen, die der Realisierbarkeit algorithmischer Kollusion skeptisch gegenüberstehen, schließen diese Möglichkeit nicht aus, s. z.B. Ulrich Schwalbe (Fn. 4), 14 Journal of Competition Law \& Economics 568, 570, 592, 596 (2018).

66 S.o. III.1. 
Unternehmen. ${ }^{67}$ Schließlich stellt die Studie Überlegungen zu der Frage an, wie Kartellbehörden die relevanten Informationen über die genannten Punkte erhalten können. Die Überlegungen reichen von den traditionellen kartellbehördlichen Untersuchungsbefugnissen bis hin zu der Frage, ob die für die Kartellaufdeckung eingesetzten Screening-Methoden auch für die Aufspürung algorithmischer Kollusion fruchtbar gemacht werden könnten. Wichtig sind auch die Überlegungen zur Art und Weise, wie Algorithmen analysiert werden können, wobei die in diesem Zusammenhang angestellten Überlegungen mangels einschlägigen Fallrechts Pioniercharakter haben. ${ }^{68}$ Grenzen werden durch die Unterscheidung zwischen "deskriptiven" und "Black-Box"-Algorithmen gezogen, wobei sich die ersteren dadurch auszeichnen, dass sie von Menschen interpretiert werden können, während das bei einer "Black-Box" nicht mehr möglich ist. ${ }^{69}$ Wenn der "Black-Box"-Charakter eine nähere Untersuchung des Algorithmus unmöglich macht, besteht immer noch die Möglichkeit, den Algorithmus zu testen, entweder in realen Bedingungen oder in einer "Sandbox". Der Test soll dann zeigen, ob der Algorithmus eine Tendenz zur Kollusion hat. ${ }^{70}$ Insbesondere bei Deep Learning-Algorithmen wird ein Test häufig die einzige realistische Möglichkeit sein, mehr über die Funktionsweise des Programms zu erfahren. ${ }^{71}$

\section{Ausweg: Beobachtungs- und Interventionspflichten}

Die vorstehenden Ausführungen haben die Schwierigkeiten deutlich gemacht, die sich bei der kartellrechtlichen Erfassung algorithmischer Kollusion stellen. Die Begriffe Vereinbarung und abgestimmte Verhaltensweise knüpfen traditionell an menschliches Verhalten an. Es bereitet deshalb keine besonderen Schwierigkeiten, Konstellationen zu beurteilen, in denen Algorithmen ein bloßes Instrument sind, dessen sich Menschen bedienen. Das Kartellrecht muss aber bereits jetzt eine Antwort auf die Frage geben, wie mit Situationen umzugehen ist, in denen das Band zwischen Mensch und Maschine länger und lockerer, ja durch Künstliche Intelligenz möglicherweise gänzlich durchtrennt wird.

67 Autorité de la concurrence / Bundeskartellamt (Fn. 36), S. $62 \mathrm{ff}$.

$68 \mathrm{Ibid}$., S. $65 \mathrm{ff}$.

69 Ibid., S. $11 \mathrm{ff}$.

70 Ibid., S. $70 \mathrm{ff}$.

71 Picht/Loderer, Framing Algorithms: Competition Law and (Other) Regulatory Tools, 42 World Competition 391, 414 (2019). 
Ausgangspunkt für eine Lösung sollte die Tatsache sein, dass im Unterschied zum Zivil- und Gesellschaftsrecht die Normadressaten des Kartellrechts nicht die (natürlichen und juristischen) Personen, sondern die Unternehmen sind. Nach der Rechtsprechung des Europäischen Gerichtshofs stellt sich ein Unternehmen "als eine einheitliche, einem selbständigen Rechtssubjekt zugeordnete Zusammenfassung personeller, materieller und immaterieller Faktoren dar, mit welcher auf die Dauer ein bestimmter wirtschaftlicher Zweck verfolgt wird". ${ }^{72}$ Zum Unternehmen gehören also nicht nur die Personen, sondern auch die "materiellen und immateriellen Faktoren", d.h. auch die Maschinen und die auf ihnen laufenden Algorithmen. ${ }^{73}$ Im Mittelpunkt sollte deshalb die Verantwortung der Unternehmen für ihre Maschinen stehen, die auch eine Pflicht zur Beobachtung und Intervention umfasst. ${ }^{74}$ Wer selbstlernende Computersysteme am Markt einsetzt, ist verpflichtet, diese ständig zu überwachen..$^{75}$ Erweist sich, dass sich die Algorithmen nicht nur "mit wachem Sinn"76 an die Konditionen von Wettbewerbern anpassen, sondern eine kollusive Strategie entwickeln, hat das Unternehmen einzuschreiten. ${ }^{77}$ Eine Verteidigung

72 EuGH, 13.7.1962, Verb. Rs. 17 und 20/61 - Klöckner Werke AG und Hoesch/Hohe Behörde, ECLI:EU:C:1962:30, S. 687.

73 Versteht man den Unternehmensbegriff in diesem Sinn umfassend, ist es nicht erforderlich, für die Zurechnung von Maschinenhandeln die Frage zu stellen, ob selbstlernende Algorithmen als "Person" qualifiziert werden sollten, s. die Diskussion bei Käseberg/von Kalben (Fn. 55), WuW 2018, S. 2 (5). Das Markthandeln der eigenen Hard- und Software wird dann dem Unternehmen ohne weiteres zugerechnet; so auch Wolf (Fn. 50), NZKart 2019, S. 2 (6f.).

74 Zum Folgenden s. Heinemann, Algorithmenbasierte Kartelle, in: Hochreutener/ Stoffel/Amstutz (Hrsg.), 10. Freiburger Tagung zum Wettbewerbsrecht - Verfahrensrecht, staatliche Wirtschaftstätigkeit und algorithmenbasierte Kartelle, 2019, S. 53 (69 f.).

75 Für eine Überwachungspflicht auch Abrantes-Metz, Pricing Algorithms and Implications for Competition, 2019, S. 5, abrufbar unter: www.competitionpolicyinter national.com/pricing-algorithms-and-implications-for-competition; Pohlmann (Fn. 28), S. 648.

76 EuGH, 16.12.1975, Verb. Rs. 40 bis 48, 50, 54 bis 56, 111, 113 und 114/73 - Suiker Unie u.a./Kommission, ECLI:EU:C:1975:174, Rn. 173/174. Der "wache Sinn", mit dem man sich dem Verhalten von Wettbewerbern anpassen darf, ohne den Vorwurf einer abgestimmten Verhaltensweise befürchten zu müssen, ist ein weiteres Beispiel für die klassisch anthropozentrische Ausrichtung des Kartellrechts, genauso wie die "Fühlungnahme" (ibid.) zwischen Wettbewerbern, die einen solchen Vorwurf begründen kann.

77 Diese Forderung wird durch neuere Tendenzen des KI-Rechts bestärkt: Im Zentrum des Vorschlags der Europäischen Kommission für eine Verordnung zur Festlegung harmonisierter Vorschriften für Künstliche Intelligenz vom 21.4.2021, 
mit dem Argument, dass man keine Kenntnis vom Verhalten des Algorithmus hatte, ist unerheblich, da eine Beobachtungspflicht besteht und bei Erkennbarkeit von Kollusion eine Intervention erforderlich ist. ${ }^{78}$

Den Beobachtungs- und Interventionspflichten sollte durch Aufstellung einer Vermutung Nachdruck verschafft werden. Wird die Preisfestlegung einem Computersystem anvertraut, und kommt es sodann zu koordinierten Preiserhöhungen im Zusammenwirken mit Computersystemen anderer Unternehmen, wird das Vorliegen einer abgestimmten Verhaltensweise vermutet. ${ }^{79}$ Die Vermutung kann durch den Nachweis widerlegt werden, dass bloßes Parallelverhalten vorliegt. Hierfür ist der Behörde oder dem Gericht die Funktionsweise des Algorithmus offenzulegen. ${ }^{80}$ Eine Black Box Defence ist nicht anzuerkennen: Die Unternehmen können sich also nicht darauf berufen, dass sie aufgrund technischer Komplexität keine Kenntnis von der Funktionsweise ihrer Algorithmen haben. ${ }^{81}$ Nach dem Untersuchungsgrundsatz obliegt es im öffentlich-rechtlichen Verfahren der Behörde, zu beurteilen, ob bloßes Parallelverhalten, z.B. Einsteigen in die Preise der Konkurrenz, vorliegt, oder aber ob eine kollusive Strategie verfolgt wird. Die in der Checkliste der deutsch-französischen Algorithmen-Studie aufgeführten Gesichtspunkte bieten sich für die Beurteilung an. ${ }^{82}$

Dieser Vorschlag geht sehr viel weniger weit als die Überlegungen, ein allgemeines Anmelde- und Genehmigungssystem für Algorithmen in der digitalen Ökonomie einzuführen. Ein solcher "TÜV für Algorithmen"83

$\operatorname{COM}(2021) 206$ final, stehen Beobachtungs-, Dokumentations-, Aufsichts- und Interventionspflichten.

78 Ebenfalls unerheblich ist der Einwand, dass die Algorithmen im Zeitablauf nicht geändert worden seien, s. Zimmer (Fn. 55), S. 1004.

79 So bereits der Vorschlag von Heinemann/Gebicka (Fn. 28), 7 Journal of European Competition Law \& Practice 431, 440 (2016); skeptisch Göhsl (Fn. 23), WuW 2018, 121 (122); Monopolkommission (Fn. 14), S. 83 f. N. $246 \mathrm{ff}$.

80 Für Offenlegungsplichten in Anlehnung an Art. 22 DSGVO Picht/Loderer (Fn. 71), 42 World Competition 391, 417 (2019).

81 Vestager, Algorithms and Competition, Berlin, 16.3.2017: "[...] companies can't escape responsibility for collusion by hiding behind a computer program. [...] And businesses also need to know that when they decide to use an automated system, they will be held responsible for what it does. So they had better know how that system works", zitiert nach Autorité de la concurrence / Bundeskartellamt (Fn. 36), S. 10 Fn. 27.

82 S.o. nach Fn. 64.

83 So die plastische Bezeichnung von Hennes/Schwalbe (Fn. 61). 
wäre nicht nur unverhältnismäßig, sondern auch unpraktikabel. ${ }^{84}$ Eine widerlegliche Vermutung passt sich hingegen in das geltende Recht ein und belastet die Unternehmen nicht übermäßig. Wer die Wirkungsweise seiner Algorithmen nicht näher überwacht, schließt bewusst die Augen und nimmt Koordination mit anderen Unternehmen in Kauf, was eine zumindest bedingt vorsätzliche Abrede belegt. Was das Spannungsverhältnis zwischen Vermutungen und der Unschuldsvermutung in strafrechtlichen und strafrechtsähnlichen Verfahren (Art. 48 Abs. 1 GRCh, Art. 6 Abs. 2 EMRK) betriff, so ist anerkannt, dass solche Vermutungen mit den Justizgrundrechten vereinbar sind, wenn sie für die Effektivität des Verfahrens erforderlich sind und die Verteidigungsrechte gewahrt bleiben. ${ }^{85}$ Diese Voraussetzungen sind in vorliegendem Zusammenhang erfüllt: Der wirksame Wettbewerb ist ein hohes Schutzgut, das durch kolludierende Algorithmen gefährdet ist. Unternehmen, die solche Algorithmen einsetzen, sind am besten dazu in der Lage, die Hintergründe maschineller Koordination aufzuklären. Ihre Verteidigungsrechte sind gewahrt, weil sie durch Offenlegung der Algorithmen die Vermutung umstoßen können. ${ }^{86}$

\section{Fazit}

Das Thema der algorithmischen Kollusion hat seit 2014 kartellrechtliche Aufmerksamkeit gefunden und wird seither intensiv diskutiert. Während juristische Diskussionen häufig ihren Ausgangspunkt von realen Problemen und konkreten Fällen nehmen, ist es hier anders: In den bereits vorliegenden Fällen steht immer noch der menschliche Einfluss auf wettbewerbsbeschränkende Koordination im Mittelpunkt. Fälle rein algorithmischer Kollusion existieren noch nicht. Dennoch hat die rechtliche Auseinandersetzung bereits einen beachtlichen Komplexitätsgrad erreicht, so

84 OECD, Algorithms and Collusion: Competition Policy in the Digital Age, 2017, S. 54 Fn. 17, abrufbar unter: www.oecd.org/competition/algorithms-collusioncompetition-policy-in-the-digital-age.htm.

85 EGMR, 7.10.1988, Fall 10519/83 - Salabiaku/Frankreich, Rn. 28.

86 Der hier gemachte Vorschlag bezieht sich auf die Verantwortlichkeit der Unternehmen, die Algorithmen einsetzen. Das Verhalten externer Dienstleister wird ihnen unter bestimmten Voraussetzungen zugerechnet, s. EuGH, 21.7.2016, C-542/14 - VM Remonts, ECLI:EU:C:2016:578. Zur Frage, unter welchen Voraussetzungen externe Anbieter von Algorithmen, also Drittpersonen, selber haften, s. Monopolkommission (Fn. 14), S. 84 ff. N. 252 ff., mit rechtspolitischer Forderung nach Einführung einer besonderen Haftung von IT-Dienstleistern, die Preisalgorithmen zum Zweck der Wettbewerbsbeschränkung anbieten. 
dass man ausreichend gewappnet ist, sollte es zu einschlägigen Fällen kommen.

Aus dem existierenden Fallrecht lassen sich bereits kleinere Schlussfolgerungen ziehen: Wie aus dem britischen Posterfall erhellt, ist es zur Erzielung von Kollusion nicht erforderlich, dieselbe Software benutzen. ${ }^{87}$ Außerdem sind tatbestandsmäßige Beschränkungen auch in Form von "Teilabreden" möglich. So programmierten die Partner des Posterkartells ihre Preissoftware durchaus so, dass die Verkaufspreise Dritter unterboten werden sollten. Für ihr Verhältnis untereinander setzten sie die Preisunterbietungsregel aber durch Eintrag des Partners auf einer ignore list außer Kraft. ${ }^{88}$ Dies zeigt, dass Algorithmen ambivalent funktionieren können, nämlich kompetitiv in einer Hinsicht, kollusiv in einer anderen. Für eine tatbestandsmäßige Vereinbarung reicht es aus, wenn der Wettbewerb zwischen mindestens zwei Unternehmen beschränkt wird. Eine Abdeckung des gesamten Markts ist nicht erforderlich. Schließlich zeigt der Luxemburger Webtaxi-Fall, dass eine tatbestandsmäßige Kollusion nicht gleichbedeutend ist mit einem illegalen Kartell. Es kann die Effizienzrechtfertigung greifen.

Darüber hinaus bleibt viel zu klären. Der Europäische Gerichtshof hat im Eturas-Entscheid ausgeführt, dass die Beteiligung eines Unternehmens "an einer Abstimmung der Verhaltensweisen nicht allein aus der Existenz der technischen Beschränkung [...] abgeleitet werden" dürfe, sondern dass eine gesonderte Billigung erforderlich sei. ${ }^{89}$ Kenntnis und Billigung scheinen für die digitalen Fälle der Zukunft nicht die richtigen Kategorien zu sein. Das anthropozentrisch interpretierte Kriterium der "Kenntnis" ist durch eine unternehmensorientierte (negotiozentrische) Sichtweise unter Einbeziehung der Maschinen zu ergänzen. Dem für eine Vereinbarung traditionellerweise geforderten meeting of the minds sollte ein meeting of the codes an die Seite gestellt werden. ${ }^{90}$ Die hierzu erforderlichen Erkenntnisfortschritte sind nur durch enge Einbeziehung der computer science zu erreichen. ${ }^{91}$ Der gewaltige Nutzen der Algorithmen ist zu würdigen, ihr Einsatz zur Beschränkung des Wettbewerbs aber zu kontrollieren. Die

87 S.o. II.1.

88 Ibid.

89 EuGH - Eturas (Fn. 25), Rn. 45

90 Alternative Formulierung in OECD (Fn. 84), S. 39: "meeting of algorithms".

91 Käseberg/von Kalben (Fn. 55), WuW 2018, S. 2 (6f.); Marty, Algorithmes de prix, intelligence artificielle et équilibres collusifs, Revue Internationale de Droit Economique 2017/2, S. 83 (109); Poblmann (Fn. 28), S. 655. 
Algorithmenbasierter Handel und Kartellverbot

machinery question von David Ricardo bedarf auch auf dem Gebiet des Kartellrechts einer zeitgemäßen Antwort. 